\title{
Médecine du travail: quel avenir pour les médecins d'entreprises non titulaires de la spécialité?
}

\author{
B. Thorens
}

\section{Bref rappel historique}

Dans un article paru en 1998 dans le Bulletin des médecins suisses [1], nous avions abordé la situation de la médecine du travail en Suisse et les modifications légales récentes qui en règlent l'exercice.

Rappelons que depuis le début de l'année 2000 déjà, les diverses entreprises de notre pays sont censées avoir procédé à une analyse de risques leur permettant de se conformer aux modifications apportées en 1996 à l'Ordonnance sur la prévention des accidents et des maladies professionnelles (OPA), selon lesquelles les entreprises qui présentent des «dangers particuliers» doivent faire appel à des médecins du travail. La directive 6508, publiée par la Commission fédérale de coordination de la sécurité au travail (CFST), règle les modalités de cet «appel aux médecins du travail et autres spécialistes de la sécurité au travail» (abrégée ci-après par «directive MSST»).

Cette démarche qui a donc débuté il y a 6 ans a pris du retard et de nombreuses entreprises ne l'ont pas encore menée à terme. Par contre, plusieurs «solutions de branches professionnelles» ont déjà été validées par la CFST. Dans de nombreux cas, ces solutions imposent le recours à des médecins du travail.

\section{Quelles conséquences pour les médecins d'entreprises?}

Les organes d'exécution, à savoir la Suva pour la Loi sur l'assurance-accident, le SECO et les inspectorats fédéraux et cantonaux pour la Loi sur le travail, ont commencé à contrôler l'application de la directive MSST dans les entreprises. Une de leur tâche consiste à vérifier si les médecins du travail qui doivent être engagés par les entreprises bénéficient bien de la formation requise. Si tel n'est pas le cas, l'entreprise concernée sera invitée à se mettre en conformité en remplaçant le médecin qui ne répond pas aux exigences légales.
Il est donc d'une extrême importance que les médecins actifs au sein des entreprises soient bien au clair sur le niveau de qualification dont ils doivent faire preuve, sous peine de devoir dans certains cas abandonner leur poste.

\section{Comment les entreprises sont-elles classées au niveau des dangers?}

La Directive MSST 6508 de la CFST distingue 3 catégories d'entreprises:

1. Entreprises ne présentant pas de danger particulier:

Pour cette catégorie, au sein de laquelle on trouve une majorité d'entreprises de services, il n'existe aucune obligation de faire appel aux spécialistes de la sécurité au travail.

2. Entreprises présentant des dangers particuliers de faible ampleur:

Comme son nom l'indique, il s'agit d'entreprises de niveau de risque intermédiaire. L'appel à des spécialistes de la sécurité au travail (le cas échéant un médecin du travail) est obligatoire uniquement pour évaluer le risque et arrêter les mesures à prendre. Mais il est naturellement envisageable qu'une telle entreprise juge nécessaire de s'adjoindre durablement un médecin du travail.

3. Entreprises présentant des dangers particuliers:

Cette catégorie regroupe les entreprises au sein desquelles existent des risques significatifs, voire élevés, d'accidents et de maladies professionnelles. Elles sont tenues de s'adjoindre des spécialistes de la sécurité au travail qualifiés et de définir les tâches qui leur incombent.

On retiendra ainsi qu'en matière d'application stricte de la Directive MSST, les entreprises de la catégorie 1 n'ont aucune obligation de s'adjoindre un médecin du travail. Celles de la catégorie 2 doivent au minimum avoir consulté un spécialiste de la sécurité (le cas échéant un médecin du travail) lors de l'élaboration de leur 
concept de sécurité et peuvent dans certains cas juger nécessaire de s'adjoindre durablement un tel spécialiste. Enfin, celles de la catégorie 3 doivent démontrer qu'elles disposent de la collaboration permanente des spécialistes de la sécurité nécessaires (notamment médecins du travail) ou avoir établi de manière irréfutable que cette présence n'est pas indispensable.

\section{Qu'est-ce qu'un «médecin du travail»?}

Jusqu'à présent, la définition du «médecin du travail» figurait dans l'Ordonnance sur les qualifications des spécialistes de la sécurité au travail (Oqual) édictée en 1996 par l'Office fédéral des assurances sociales (OFAS). Est réputé médecin du travail le médecin qui possède le titre de médecin du travail selon l'Ordonnance du 17 octobre 2001 sur la formation postgraduée et la reconnaissance des diplômes et des titres de formation postgraduée des professions médicales.

Ces exigences sont celles qui permettent actuellement d'obtenir le titre de spécialiste FMH en médecine du travail à l'issue d'une période de formation postgraduée de 5 ans [2].

En principe donc, seuls des médecins au bénéfice de ce titre de spécialiste ou d'une formation jugée équivalente seraient habilités à fonctionner comme «médecin du travail» dans une entreprise tenue d'appliquer la directive MSST.

Suite à l'entrée en vigueur des accords bilatéraux avec l'Union Européenne, la description de la formation postgraduée pour les médecins disparaît de l'Ordonnance sur les qualifications. Comme depuis le $1^{\text {er }}$ juin 2002, les titres de spécialiste sont conférés par la Confédération, l'Ordonnance révisée sur la libre circulation renvoie directement au titre de spécialiste en médecine du travail. Ces nouvelles dispositions légales peuvent être consultées dans le site web de la FMH www.fmh.ch au chapitre «Formation postgraduée».

\section{Manque en médecins du travail: recherche d'une solution}

Nous relevions en 1998 déjà le manque relatif de spécialistes en médecine du travail. La situation n'a pas changé depuis, le nombre de porteurs du titre restant stable aux environs de 65 et seuls une vingtaine d'entre eux disposant d'une certaine disponibilité pour de nouvelles tâches au sein des entreprises.

Bien que les analyses de risque livrées à ce jour ne fassent pas apparaître le besoin d'un grand nombre de médecins du travail, de nombreuses entreprises suisses recourent ou devront recourir au service de médecins non spécialistes pour répondre à leurs besoins.

Il s'agissait donc de trouver une solution pragmatique au sein d'un groupe de travail piloté par l'OFAS, chargé de gérer l'application de l'Oqual et constitué de représentants des organes d'exécution (Suva et seco), de la CFST et des sociétés de spécialistes en médecine et sécurité au travail.

Sous l'égide de la FMH, les trois sociétés de spécialistes les plus directement concernées, la Société suisse de médecine du travail (SSMT), de médecine générale (SSMG) et de médecine interne (SSMI) ont élaboré un concept visant à gérer au mieux cette situation en conciliant les impératifs de qualité des prestations médicales fournies, les intérêts des médecins actuellement actifs au sein des entreprises ou susceptibles de le devenir encore dans un très proche avenir et les nouvelles dispositions légales en vigueur. Ce concept a été formellement accepté par le Comité central de la FMH lors de sa session de mars 2002 à l'attention des organes d'exécution que sont la Suva, le SECO et la CFST. Il se présente comme suit:

Les médecins non titulaires de la spécialité en médecine du travail ou d'une formation jugée équivalente (Oqual), exerçant une fonction relative à la médecine du travail au sein d'entreprises dans le cadre de l'OPA sont autorisés à poursuivre celle-ci ou à accepter de nouveaux mandats dans des entreprises de même type, aux conditions suivantes:

- être en fonction au $1^{\text {er }}$ janvier 2000 lorsqu'il s'agit d'entreprises présentant des dangers particuliers (art. 2.3 de la directive CFST 6508);

- avoir débuté leur activité avant le $1^{\text {er }}$ janvier 2004 lorsqu'il s'agit d'entreprises présentant des dangers particuliers de faible ampleur (art. 2.2 de la directive CFST 6508);

- suivre un cours de formation de base en médecine du travail;

- s'astreindre à la formation continue complète en médecine du travail dès 2004. La fréquentation des 2 modules du cours de l'ETHZ/UNIL pourra être validée comme formation continue pour l'année concernée. 


\section{Conséquences pratiques pour les médecins désirant satisfaire aux exigences de la directive MSST}

\section{Cours de formation de base}

Ce cours comprend 2 parties:

- 6 jours de formation théorique. Ils apporteront aux participants les bases essentielles en médecine du travail.

- Fréquentation de 2 modules de 2 jours du cours de l'ETHZ/UNIL «Travail + santé» au choix du candidat.

- Lieux: centralisés, pour la Suisse romande et pour la Suisse alémanique.

- Dates:

1. formation théorique de 6 jours répartie sur $3 \times 2$ jours (vendredi et samedi) au cours de l'année 2003;

2. modules du cours ETHZ/UNIL: selon le programme de ceux-ci, à suivre dans les 3 ans (2004-2006).

- Coûts: Le montant de participation au cours de base de 6 jours et à deux modules du cours «Travail + santé» se montera à Fr. 3000 environ.

\section{Attestation}

A l'issue de la fréquentation des deux parties du cours de base, une attestation de la FMH sera remise aux participants.

\section{Formation continue}

En vertu des accords passés entre la SSMT, la SSMI et la SSMG, le montant total de crédits requis ne dépasse pas 60 points pour les médecins devant accomplir leur formation continue en médecine du travail et, respectivement, en médecine interne ou générale.

\section{Préinscription obligatoire!}

Attention! Le cours de base de $3 \times 2$ jours ne sera organisé qu'une seule et unique fois au cours $d u$ $2^{e}$ semestre 2003 et ne sera pas répété. Pour planifier son organisation il est indispensable de connaître le nombre de médecins intéressés.

Nous invitons donc les consœurs et confrères intéressé(e)s à s'inscrire jusqu'au 15 septembre 2002 à l'adresse suivante: Dr B. Thorens, Suva, Case postale, 1001 Lausanne.

\section{Renseignements complémentaires}

Des renseignements complémentaires peuvent être obtenus auprès de:

\section{SSMT}

Dr B. Thorens, Suva, Avenue de la Gare 19, 1001 Lausanne, tel. 02131080 78, e-mail: blaise.thorens@suva.ch.

\section{SSMI}

Dr C. Witz, Rue des Deux-Ponts 20, Case postale 198, 1211 Genève 8 , fax 0223290794 .

\section{SSMG}

SGAM/SSMG Secrétariat, Oberplattenstrasse 73, 9620 Lichtensteig, tel. 07198866 40, fax 07198866 41, e-mail: sgam@hin.ch.

FMH (pour les questions juridiques) $\mathrm{M}^{\mathrm{e}}$ HP. Kuhn, Secrétaire général adjoint, FMH, Case postale 293, 3000 Berne 16, tel. 03135911 11, fax 03135911 12, e-mail: fmhrecht@hin.ch.

\section{Références}

1 Thorens B. La médecine du travail en Suisse: par qui est-elle pratiquée? Bull Méd Suisses 1998; 79(19):851-2.

2 Règlement de formation postgraduée pour l'obtention du titre de spécialiste FMH en médecine du travail. 